ISSN 2525-4804

\title{
DENSIDADE E POROSIDADE DE UM PLINTOSSOLO HÁPLICO SUBMETIDO A DIFERENTES TIPOS DE USO
}

\author{
Luíz Paulo Figueredo Benício; ${ }^{1}$ Denyse Sousa Nascimento²; João Paulo Prado de Melo²
}

\section{RESUMO:}

Os Plintossolos são uma parcela significativa dos solos agrícolas do Estado do Tocantins. Dessa forma, é importante entender o comportamento desses solos quando submetidos a diferentes tipos de uso. O trabalho foi conduzido no município de Couto Magalhães-TO em um Plintossolo Háplico. Foi adotado o delineamento inteiramente ao acaso no esquema fatorial $3 \times 2$ três usos (mata nativa, plantio direto e plantio convencional) e duas profundidades $(0-20 \mathrm{~cm}$ e $20-40 \mathrm{~cm})$. Os usos do solo foram plantio direto, plantio convencional e mata nativa. Foi avaliado a porosidade do solo, densidade do solo e a correlação entre estes atributos. O sistema de plantio convencional aumenta a porosidade do solo e consequentemente reduz a densidade em ambas profundidades. Já o sistema de plantio direto resultou em um maior adensamento do solo, porém em valores que não limitam o cultivo, mostrando que os dois sistemas são viáveis para o cultivo neste tipo de solo.

Palavras-chave: Física do solo, plantio convencional, plantio direto, solos do Tocantins.

\section{DENSITY AND POROSITY OF A HAPLASTIC PLINTHOSSOL SUBMITTED TO DIFFERENT USE}

\section{ABSTRACT:}

Plinthossols are great part of agricultural soils of Tocantins, in this sense is very important understand the modifications promoted in these soils by agricultural use. The experiment had made in Couto Magalhães in a Haplistic Plinthosol. Was adopted the casuistic design in a 3x2 factorial scheme three uses (native vegetation, conventional tillage and no-tillage) and two depth $(0-20 \mathrm{~cm}$ e $20-40 \mathrm{~cm})$ The soil uses had conventional tillage, no-tillage, and native forest. The conventional planting system increases soil porosity and consequently reduces density at both depths. The no-tillage system, on the other hand, resulted in a greater density of the soil, but in values that do not limit cultivation, showing that both systems are viable for cultivation in this type of soil.

Keywords: soil physics, conventional tillage, no-tillage, Tocantins soils.

\footnotetext{
${ }^{1}$ Dsc. Em Solos e Nutrição de Plantas pela Universidade Federal de Viçosa. E-mail: luizpaulo.figueredo@gmail.com

${ }^{2}$ Agroll Consultoria
} 


\section{INTRODUÇÃO}

No mundo mais de 700 milhões de pessoas não têm comida suficiente para levar uma vida saudável e ativa (World Health Organization, 2018, FAO, IFAD, UNICEF, WFP, e WHO, 2019). Para manter a segurança alimentar de uma população em constante crescimento será necessário um grande aumento da produção de alimentos nos próximos anos, e o grande desafio é realizar esse aumento sem abertura de novas áreas. Diante dessa necessidade sistemas mais intensivos de produção vêm sendo adotados (Saath e Fachinello, 2018), permitindo que áreas antes consideradas marginais sejam incorporadas ao sistema produtivo, sobretudo no Brasil.

O Tocantins é um estado brasileiro localizado na Região Norte que nos últimos anos tem ganhado notoriedade por sua produção agrícola. Dados da Conab (2020) mostram que o estado produziu 5,8 milhões de toneladas de grãos, cultivados em 1,5 milhões de hectares, e uma produtividade média de $3.752 \mathrm{~kg} \cdot \mathrm{ha}^{-1}$. Dentre as classes de solos presentes no estado do Tocantins os Plintossolos (Pétricos, Háplicos e Argilúvicos) ocupam 11,1\% do território, sendo a terceira classe de solo, em área (Seplan, 2012). Estes solos são formados sob condições de restrição à percolação de água, e apresentam um horizonte plíntico ou litoplíntico, e uma má drenagem. São solos fortemente ácidos e com baixa atividade de argila (Santos et al., 2018). Mesmo com características que aparentemente limitam o cultivo, muitas áreas de produção de grãos estão sobre esta classe de solo, principalmente na região central do estado.

Trabalhos conduzidos por Nikkel e Lima (2019) mostram que a presença de concreções no solo afeta o desenvolvimento da soja. Os autores atribuem este efeito a possíveis limitações ao crescimento radicular causado pelas concreções, e a um menor armazenamento de água, quando comparados a um solo sem a presença de concreções em seu volume total.

Sistemas de cultivos conservacionistas como cultivo mínimo, plantio direto ou integração lavoura-pecuária são importantes ferramentas para promover incrementos na produtividade de áreas como por exemplo os Plintossolos do Tocantins (Almeida et al., 2020), pois o acúmulo de material orgânico no solo melhora a saúde do sistema através do aumento de várias propriedades físico-químicas, melhorando as condições de cultivo inclusive em solos com propriedades físicas limitantes com presença de laterita (Jain e Kalamdhad, 2020).

$\mathrm{Na}$ literatura há poucos relatos da melhoria das condições de cultivos em Plintossolos, todavia ainda encontram-se resultados importantes. Parker (2016), avaliando diferentes sistemas de cultivo em áreas com Plintossolos, mostrou que o plantio direto na sucessão milho-feijão caupi promoveu melhorias significativas em atributos do solo como porosidade, densidade, estabilidade de agregados e condutividade hidráulica. Silva et al. (2012), trabalhando com sistemas agroflorestais (SAFs) sobre um Plintossolo Háplico, verificaram que mesmo dentro de um sistema complexo como os SAFs a variação da espécie cultivada, sobretudo às leguminosas promovem mudanças nos atributos físicos do solo, melhorando o ambiente para crescimento radicular.

Tendo em vista que os Plintossolos representam uma parcela importante dos solos do Estado do Tocantins, torna-se necessário compreender melhor o comportamento destes solos sobre diferentes usos e manejos. O presente trabalho teve por objetivo avaliar as alterações de atributos físicos como densidade e porosidade em um Plintossolo Háplico sob cultivo de soja no estado do Tocantins.

\section{MATERIAL E MÉTODOS}


O trabalho foi realizado na Fazenda Alvorada localizada no município de Couto Magalhães-TO. A propriedade é localizada nas coordenadas $8^{\circ} 34^{\prime} 05.8^{\prime \prime S} 4^{\circ} 05^{\prime} 48.3^{\prime \prime O}$ a uma altitude de $150 \mathrm{~m}$. O clima segundo a classificação de Köppen, é Aw, mesotérmico úmido, com verões quentes e invernos secos.

O trabalho foi desenvolvido em um Plintossolo Háplico textura média (Santos et al., 2018). A fazenda possui áreas cultivadas sob o sistema de plantio convencional (PC), plantio direto (PD) e mata nativa (MN). $\mathrm{Na}$ área de $\mathrm{PC}$, o solo é cultivado com soja há três safras, onde o preparo do solo é realizado com duas gradagens, usando grade aradora de discos com 28 polegadas. $\mathrm{Na}$ área sob sistema de PD é utilizada a sucessão milheto-soja há quatro anos. A área de mata nativa é composta por vegetação de Cerrado preservado sem interferência antrópica. As amostras foram coletadas no período entressafra (setembro) após o início das chuvas. As amostras foram coletadas de forma aleatória tanto no PC quanto no PD, sem considerar a localidade das linhas de plantio.

Para realização do trabalho adotou-se o delineamento inteiramente ao acaso no esquema fatorial $3 \times 2$ (três tratamentos e duas profundidades) com 10 repetições. Os tratamentos foram constituídos de áreas sob diferentes tipos de uso (MN, PC e PD). Os parâmetros físicos avaliados foram densidade do solo (DS) e porosidade total (PT) nas profundidades de $0-10$ e $10-20 \mathrm{~cm}$.

Para determinação da DS e PT adotou-se o método do anel volumétrico descrito por Embrapa (1997) com cilindros de aço inox. Previamente à coleta, cada cilindro foi mensurado para que fosse conhecido o volume coletado em cada ponto. Para a realização da retirada das amostras indeformadas o anel foi inserido no solo com um amostrador tipo castelo com um auxílio de uma marreta, e os excessos de solo foram retirados com canivete. Em uma das faces do cilindro foi colocado um pedaço do tecido preso por um elástico a fim de reduzir as perdas do material.

Para determinação da PT os cilindros foram colocados em uma bandeja com água por \pm 12 horas para os poros ficarem totalmente saturados com água. Após saturadas as amostras foram pesadas em balança com aproximação 0,001g. Logo em seguida foram colocadas em estufa por 48 horas $105{ }^{\circ} \mathrm{C} \pm 5{ }^{\circ} \mathrm{C}$, sendo novamente pesadas após o período de secagem. A PT e DS foram obtidas pelas equações 1 e 2 respectivamente.

$$
\begin{array}{ll}
\mathrm{PT}(\%)=\frac{P u-P s S x(100)}{\mathrm{Vt}} & \text { Eq. } 1 \\
\mathrm{DS}=\frac{P S S}{\mathrm{Vt}} & \text { Eq. } 2
\end{array}
$$

Onde: $\mathrm{Pu}=$ Peso do solo úmido após 12 horas de saturação. Pss $=$ Peso de solo seco. $\mathrm{Vt}=$ volume total do cilindro.

Ainda, em cada uma das áreas avaliadas foram coletadas amostras para determinação dos teores de matéria orgânica pelo método WalkleyBlack (Embrapa, 1997).

Os dados referentes à análise física e de matéria orgânica foram submetidos à análise de variância, e as médias comparadas pelo teste Tukey com nível de significância de 5\%, para as análises estatísticas foi utilizado o software SISVAR (Ferreira, 2014). Também foram realizados testes de correlação entre a PT e DS, e entre a DS e os teores de MOS.

\section{RESULTADOS E DISCUSSÃO}

A tabela 1 mostra o resumo da análise de variância dos atributos PT e DS. O resultado mostra que houve diferenças entre os atributos avaliados somente para a fonte de variação uso do solo, não havendo diferenças entre as profundidades avaliadas, e nem interação significativa entre uso e profundidade. 
Tabela 1. Resumo da análise de variância da DS e PT em função de diferentes usos do solo em diferentes profundidades.

\begin{tabular}{cccc}
\hline \multirow{2}{*}{ F.V. } & GL & \multicolumn{2}{c}{ Quadrados Médios } \\
\cline { 3 - 4 } & & DS & PT \\
\hline Uso & 2 & $0,16^{*}$ & $341,69 *$ \\
\hline Profundidade & 2 & $0,011 \mathrm{~ns}$ & $5,97 \mathrm{~ns}$ \\
\hline Uso x Profundidade & 2 & $0,020 \mathrm{~ns}$ & $21,04 \mathrm{~ns}$ \\
\hline Resíduo & 54 & 0,032 & 70,93 \\
\hline
\end{tabular}

* Diferença significativa $\mathrm{p}<0,05$ pelo teste $\mathrm{f}$. ns não significativo.

Os resultados de DS são mostrados na Figura 1. Para este atributo houve diferenças entre os usos do solo, onde a $\mathrm{MN}$ foi o uso que apresentou menor valor de DS, diferindo apenas do PD, uso com as maiores médias de DS.
Quanto as diferentes profundidades, não houve diferença entre os valores de DS, indicando que de $0-20 \mathrm{~cm}$ as áreas em estudo apresentam uma uniformidade em suas densidades.

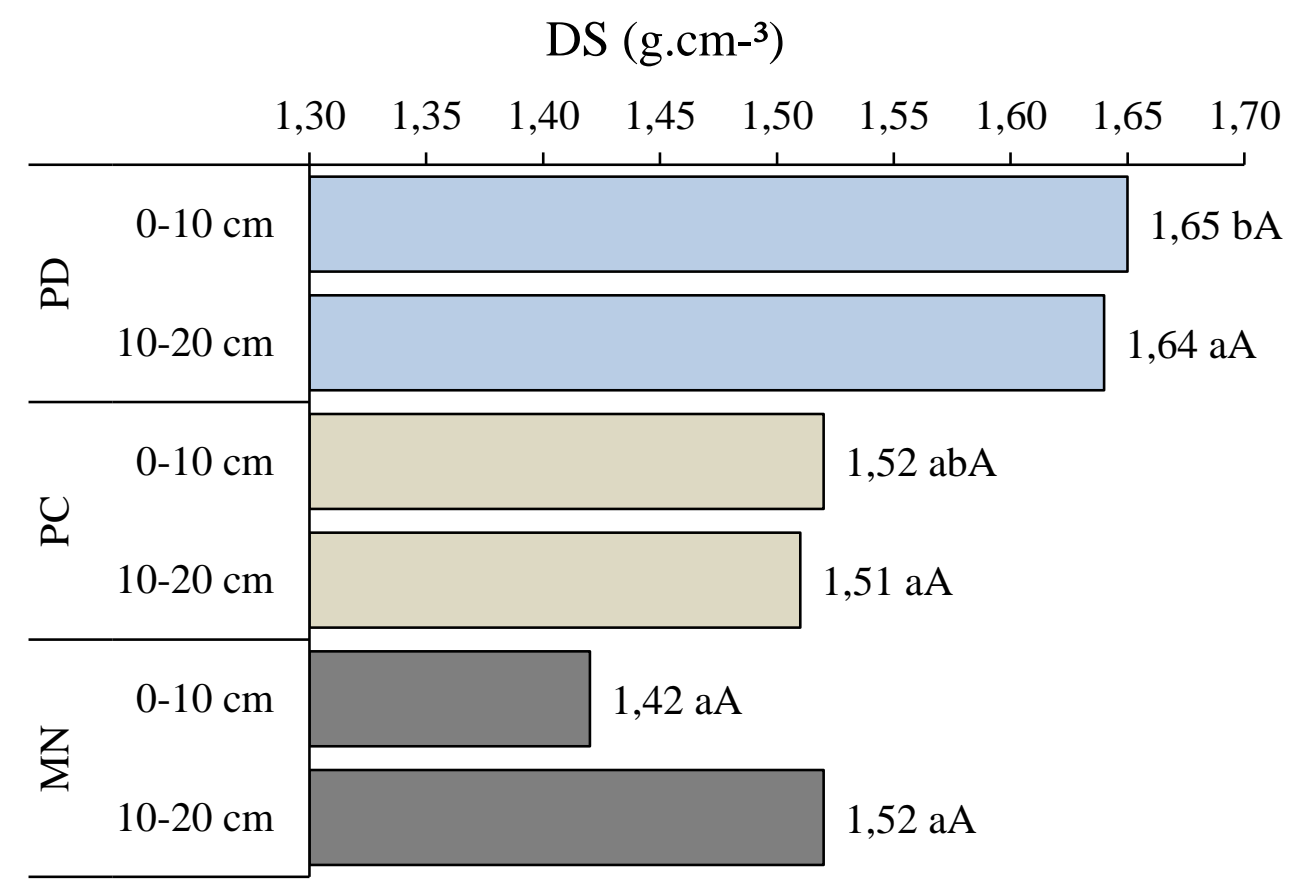

Figura 1. Densidade do solo em duas profundidades em função de diferentes tipos de uso do solo. Letras minúsculas comparam diferenças entre os sistemas para cada profundidade, a $5 \%$ pelo teste Tukey e Letras maiúsculas comparam diferenças entre as duas profundidades de cada sistema, a 5 $\%$ pelo teste Tukey.

Os resultados obtidos podem ser explicados pelo fato de que a MN é um sistema ainda não alterado, mantendo suas características naturais, já para o PD e PC o tráfego de máquinas e implementos foram capazes de promover o adensamento do solo e consequentemente a sua densidade. O tráfego de máquinas e implementos agrícolas são um dos maiores responsáveis pelo adensamento do solo em sistemas agrícolas 
(Hajabbasi et al.,1997, Lima et al., 2011, Júnnyor et al., 2019, Esteban et al., 2019).

$\mathrm{O}$ fato da área de PD ter apresentado maiores valores de densidade pode ser explicado pelo fato de que pelo menos a quatro anos há o tráfego de máquinas sem o revolvimento no solo, promovendo o aparecimento de camadas compactadas, ao passo que no solo sob PC há um revolvimento do solo anualmente, aumentando assim sua porosidade e consequentemente reduzindo a densidade, conforme relatado por Marcolan et al. (2007).

Os valores de DS encontrados em todas as áreas estão dentro dos limites aceitos para o desenvolvimento das raízes das plantas segundo
Reinert et al. (2008). Os autores, testando diferentes plantas, verificaram que valores de DS abaixo de $1,85 \mathrm{~g} / \mathrm{cm}^{3}$ não afetam o desenvolvimento radicular das plantas, porém ressaltam que ocorrem variações em função da classe textural do solo.

Na Figura 2 encontram-se os resultados para a avaliação da PT. A respeito desta foi observado que na área sob PC os valores médios foram maiores seguido pela mata nativa, e diferindo da área sob PD. Estes resultados podem ser explicados pelo revolvimento do solo promovido pelos implementos no sistema de plantio convencional.

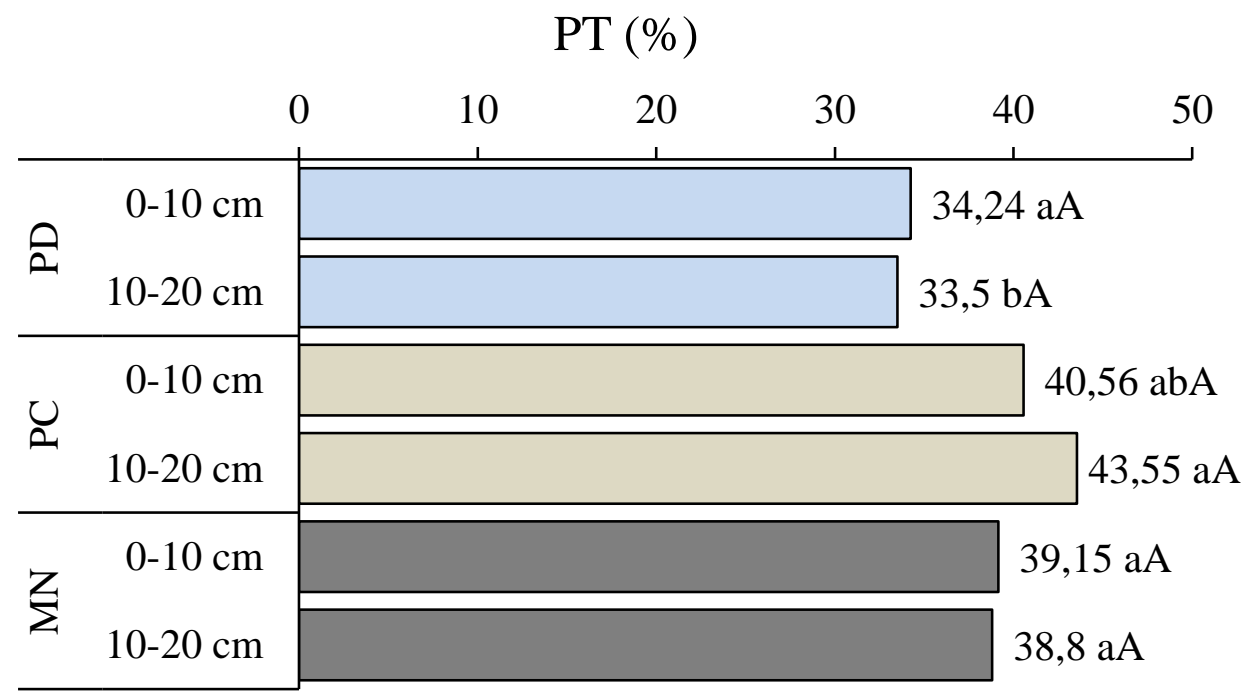

Figura 2. Porosidade total do solo em duas profundidades em função de diferentes tipos de uso do solo. Letras minúsculas comparam diferenças entre os sistemas para cada profundidade, a $5 \%$ pelo teste Tukey e Letras maiúsculas comparam diferenças entre as duas profundidades de cada sistema, a $5 \%$ pelo teste Tukey. 
A menor PT e a maior DS no solo sob semeadura direta deve-se à pressão exercida no solo pelo trânsito de máquinas e implementos, bem como pela consolidação do solo, tendo em vista a ausência quase que completa de preparo na semeadura direta (Bertol et al, 2004, Moreira et al. 2012, Rós et al., 2013).

Ao comparar os atributos físicos do solo observa-se uma grande diferença entre a $\mathrm{MN}$ e o PD, onde por se tratar de um sistema conservacionista espera-se que apresente características próximas a um sistema natural. Todavia, o tempo é fundamental para a melhoria nos sistemas, conforme relatado por (Assis e Lanças, 2005). Os autores mostram que os valores de DS de um solo sob PD se equiparou aos valores em MN após doze anos de implantação do sistema. No presente trabalho, assim como no desenvolvido por Santos et al.
(2014), os sistemas estudados não foram capazes de promover melhorias nos atributos físicos do Plintossolo.

Os teores de matéria orgânica encontrados nas áreas foram 1,56\% no PD, 1,40 $\%$ no PC e 1,38 \% em MN. Os teores de matéria orgânica encontrados nas áreas estudadas podem ser descritos como baixos conforme a classificação de Prezotti e Guarçoni (2013). De maneira geral em regiões tropicais há uma perda de carbono muito elevada, tornando o acúmulo de matéria orgânica no solo, principalmente em sistemas de cultivo (Silva e Machado, 2000).

$\mathrm{Na}$ Figura 3 pode-se observar que ocorre uma redução de DS à medida em que se aumenta a porosidade, o que é de se esperar já que a DS é a relação entre volume e massa de solo seco. Assim, quanto maior o volume ocupado por espaços porosos menor será sua densidade.

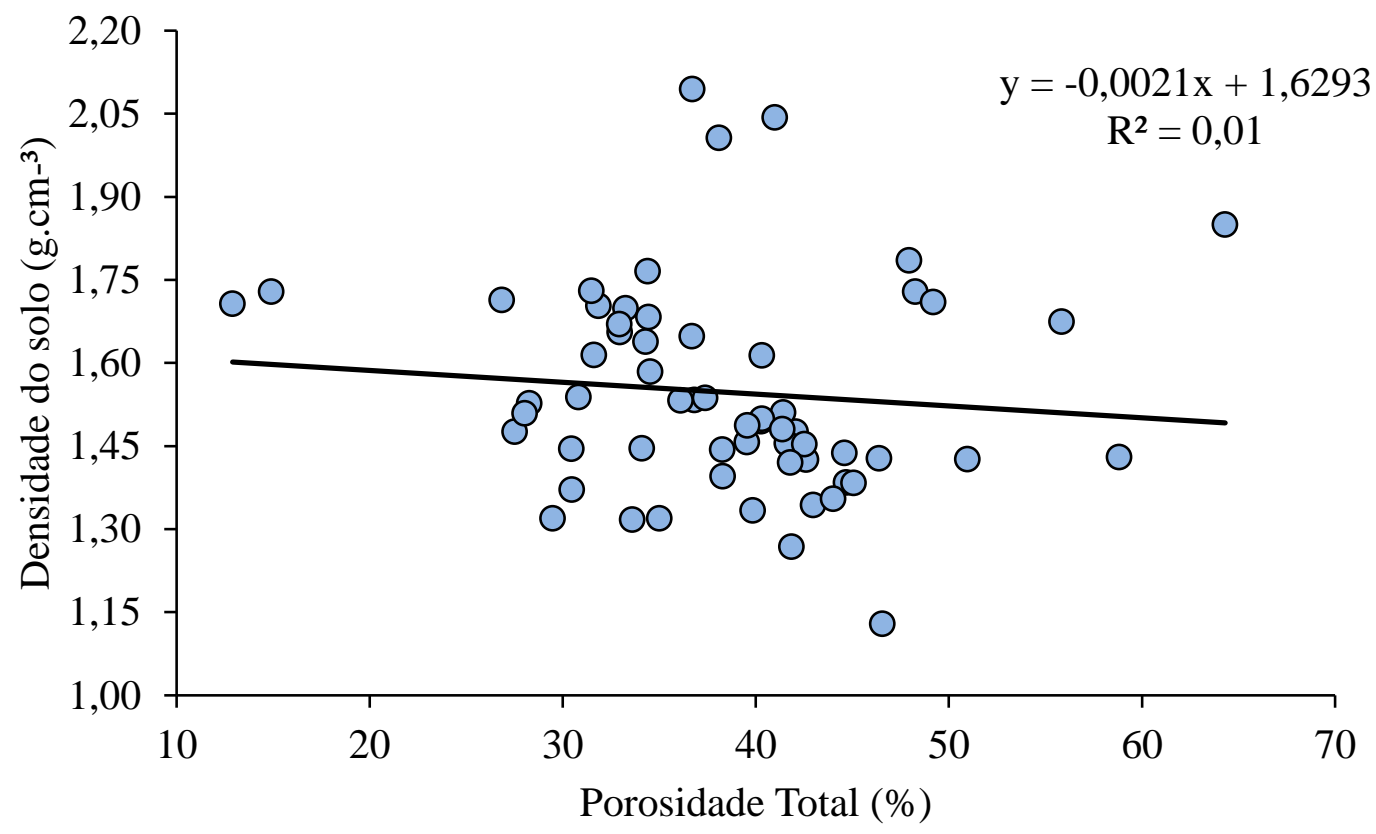

Figura 3. Correlação entre porosidade total e densidade do solo. 
A densidade de solo e porosidade são características de grande importância no estudo de propriedades físicas do solo. Estas características estão relacionadas com à estrutura e densidade de partículas sendo indicador de qualidade do solo, que são facilmente alteradas com uso e manejo do solo (Viana, 2008).

Na Figura 4 encontram-se a correlação entre os teores de matéria orgânica do solo e a DS. Os resultados obtidos mostram que não há correlação significativa entre os teores de matéria orgânica e a DS, porém ocorre uma tendência de redução de DS em função do aumento dos teores de matéria orgânica.

Aquino Lemos Filho et al. (2008) relatam uma estrita correlação entre os teores de matéria orgânica do solo e DS e informam que nem sempre ocorre uma significância, pois ambos parâmetros dependem da variabilidade espacial na área.

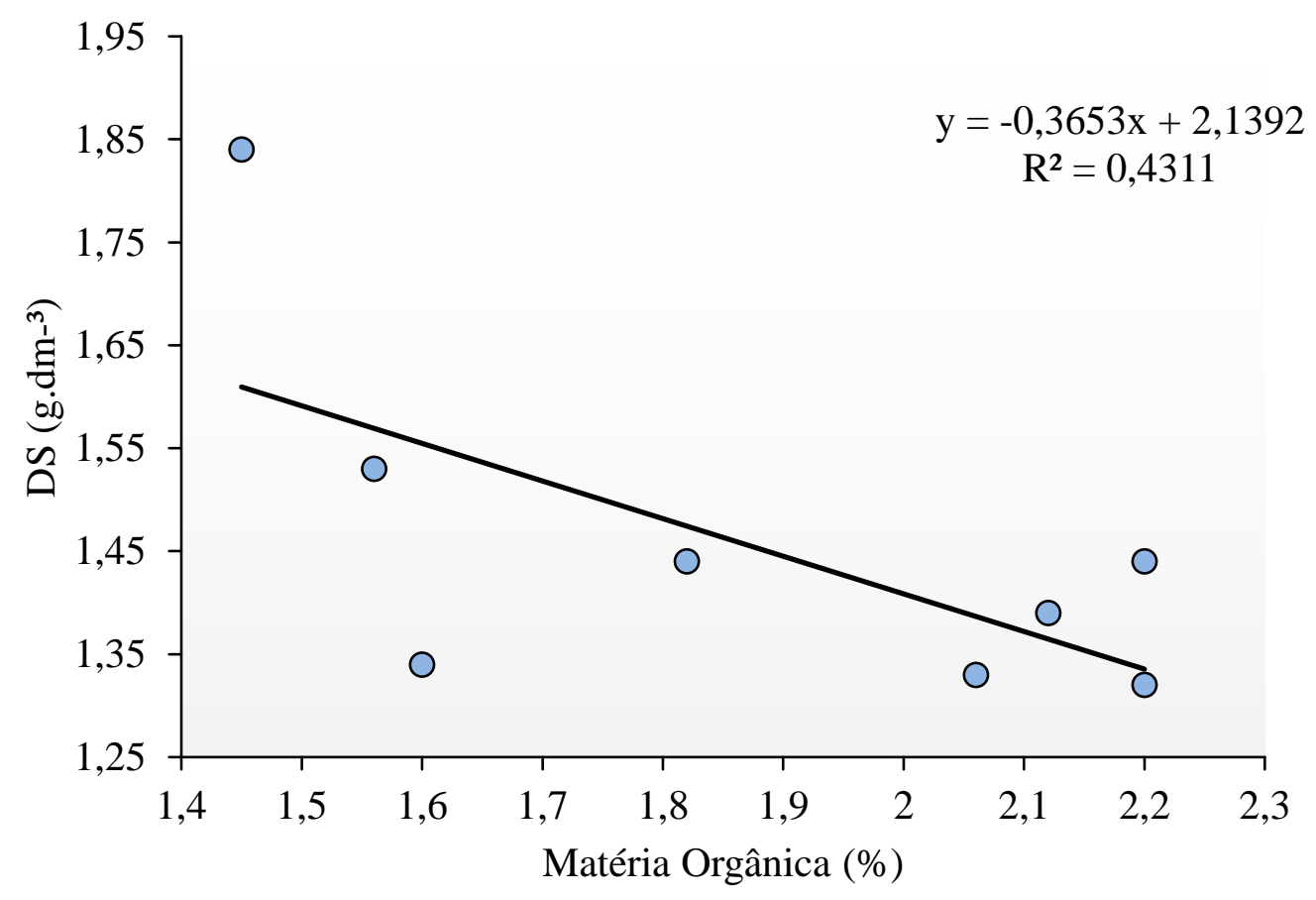

Figura 4. Correlação entre os teores de matéria orgânica do solo e densidade do solo.

\section{CONCLUSÃO}

A partir dos dados obtidos pode-se concluir que os sistemas de cultivo adotados alteram a densidade do solo em relação à sua condição natural, tanto em superfície quanto em subsuperfície, sendo o plantio direto o sistema que promove maior adensamento. Por isso, o sistema de plantio convencional, em razão de atividades operacionais que pulverizam o solo, promove um aumento na porosidade do solo em relação ao plantio direto.

Mesmo com maiores valores de densidade encontrados no sistema de plantio direto, os valores encontrados não são limitantes para o cultivo da soja. Pode-se observar também que a densidade do solo apresenta uma correlação com a matéria orgânica, onde maiores teores de matéria orgânica tendem a reduzir a densidade. Dessa forma, pode-se inferir que no 
sistema de plantio direto, com o passar dos anos, a densidade do solo pode sofrer reduções em função da matéria orgânica acumulada. Assim, fíca como sugestão um novo estudo de longa duração para avaliar melhor e validar ou não esta hipótese.

\section{REFERÊNCIAS BIBLIOGRÁFICAS}

Almeida, R. E. M., Uhlmann, A., Campos, L., \& Da Costa, R. V. (2020). Expansão agrícola em áreas de difícil manejo: cultivo em solos com cascalho. Embrapa Pesca e Aquicultura-Artigo em periódico indexado (ALICE). Disponível em: https://www.alice.cnptia.embrapa.br/bitstream/d oc/1124588/1/CNPASA-2020-abtnv.pdf

Aquino Lemos Filho, L. C., de Oliveira, E. L., de Faria, M. A., \& Bastos Andrade, L. A. (2008). Variação espacial da densidade do solo e matéria orgânica em área cultivada com cana-de-açúcar (Saccharum officinarum L.). Revista Ciência Agronômica, 39(2), 193-202.

Assis, R. L. D., \& Lanças, K. P. (2005). Avaliação dos atributos físicos de um Nitossolo Vermelho distroférrico sob sistema plantio direto, preparo convencional e mata nativa. Revista Brasileira de Ciência do Solo, 29(4), 515-522. https://doi.org/10.1590/S0100$\underline{06832005000400004}$

Bertol, I., Albuquerque, J. A., Leite, D., Amaral, A. J., \& Zoldan Junior, W. A. (2004). Propriedades físicas do solo sob preparo convencional e semeadura direta em rotação e sucessão de culturas, comparadas às do campo nativo. Revista Brasileira de Ciência do Solo, 28(1), 155-163. https://doi.org/10.1590/S0100$\underline{06832004000100015}$

Conab, Companhia Nacional De Abastecimento. Acompanhamento da safra brasileira: $1^{\circ}$ levantamento da safra $\mathbf{2 0 1 9 / 2 0 2 0}$, setembro 2020.

Disponível https://www.conab.gov.br/infoagro/safras/graos/boletim-da-safra-degraos/item/download/33275_6780e71910d3f0d 489c5f171231b65cd

Embrapa. (1997). Manual de métodos de análise de solo. Rio de Janeiro: Embrapa Solos.

Esteban, D. A. A., de Souza, Z. M., Tormena, C. A., Lovera, L. H., Souza Lima, E., Oliveira, I. N., \& Paula Ribeiro, N. (2019). Soil compaction, root system and productivity of sugarcane under different row spacing and controlled traffic at harvest. Soil and Tillage Research, 187, 60-71. https://doi.org/10.1016/j.still.2018.11.015

FAO, I., \& UNICEF. WFP and WHO (2019) 'The State of Food Security and Nutrition in the World 2019-Safeguarding against economic slowdowns and downturns'. Rome, FAO.

Ferreira, D. F. (2014). Sisvar: A computer statistical analysis system for windows version 5.6. Ciência Agrotecnologia, 38(2), 109-112. https://doi.org/10.1590/S1413-

$\underline{70542014000200001}$

Hajabbasi, M. A., Jalalian, A., \& Karimzadeh, H. R. (1997). Deforestation effects on soil physical and chemical properties, Lordegan, Iran. Plant and soil, 190(2), 301-308. https://doi.org/10.1023/A:1004243702208

Jain, M. S., \& Kalamdhad, A. S. (2020). Soil revitalization via waste utilization: Compost effects on soil organic properties, nutritional, sorption and physical properties. Environmental Technology \& Innovation, 18 , 100668. https://doi.org/10.1016/j.eti.2020.100668

Júnnyor, W. D. S. G., Maria, I. C. D., AraujoJunior, C. F., Lima, C. C. D., Vitti, A. C., Figueiredo, G. C., \& Dechen, S. C. F. (2019). 
Soil compaction on traffic lane due to soil tillage and sugarcane mechanical harvesting operations. Scientia Agricola, 76(6), 509-517. https://doi.org/10.1590/1678-992x-2018-0052 Lima, S. O., Benício, L. P. F., Borja Reis, A. F., \& Oliveira, A. G. (2011) Change in physical properties of cerrado oxisol under crop-livestock integration in the state of Tocantins, Brazil. Global Science and Technology 4(3), 1-10.

Marcolan, A. L., Anghinoni, I., Fraga, T. I., \& Leite, J. G. D. B. (2007). Recuperação de atributos físicos de um argissolo em função do seu revolvimento e do tempo de semeadura direta. Revista Brasileira de Ciência do Solo, 31(3), 571-579. https://doi.org/10.1590/S0100$\underline{06832007000300017}$

Moreira, W. H., Betioli Junior, E., Petean, L. P., Tormena, C. A., Alves, S. J., Costa, M. A. T., \& Franco, H. H. S. (2012). Atributos físicos de um Latossolo Vermelho distroférrico em sistema de integração lavoura-pecuária. Revista Brasileira de Ciência do Solo, 36(2), 389-400. https://doi.org/10.1590/S0100-

$\underline{06832012000200008}$

Nikkel, M., \& Lima, S. O. (2019). Growth and vegetative development of soybean plants in soil type Concrectionary Petric Plinthosol. Scientia Agraria Paranaensis, 351-356. https://doi.org/10.18188/sap.v18i4.22452

Parker, Bismark Quarku. (2016). The impact of tillage, cowpea-maize rotation and mulching on the physicochemical properties of a Haplic Plinthosol in Ghana. (Tese de Doutorado). Kwame Nkrumah University of Science and Technology. Kumasi, Ghana.

Prezotti, L. C.; Guarçoni, A. M. Guia de interpretação de análise de solo e foliar. Vitória, ES: Incaper, 2013. 104p.
Reinert, D. J., Albuquerque, J. A., Reichert, J. M., Aita, C., \& Andrada, M. M. C. (2008). Limites críticos de densidade do solo para o crescimento de raízes de plantas de cobertura em Argissolo Vermelho. Revista Brasileira de Ciência do Solo, 32(5), 1805-1816. https://doi.org/10.1590/S0100-

$\underline{06832008000500002}$

Rós, A. B., Tavares Filho, J., \& Cesare Barbosa, G. M. (2013). Propriedades físicas de solo e crescimento de batata-doce em diferentes sistemas de preparo. Revista Brasileira de Ciência do Solo, 37(1), 242-250. https://doi.org/10.1590/S0100-

$\underline{06832013000100025}$

Saath, K. C. D. O., \& Fachinello, A. L. (2018). Crescimento da demanda mundial de alimentos e restrições do fator terra no Brasil. Revista de Economia e Sociologia Rural, 56(2), 195-212. https://doi.org/10.1590/1234-56781806$\underline{94790560201}$

Santos, F. C., Freitas, I. C., Correchel, V., \& Carneiro, M. A. C. (2014). Qualidade física de um Plintossolo Argilúvico sob diferentes sistemas de gestão. Revista de Ciências Agrárias, 37(2), 152-161.

Santos, H. G., Jacomine, P. K. T., Dos Anjos, L. H. C., Oliveira, V. A., Lumbreras, J. F., Coelho, M. R., ... \& Cunha, T. J. F. (2018). Sistema brasileiro de classificação de solos. Brasília, DF: Embrapa.

Seplan, Secretaria do Planejamento e da Modernização da Gestão Pública. (2012). Atlas do Tocantins: subsídios ao planejamento da gestão territorial. 6. ed. rev. atu. Palmas: Seplan, 80 p.

Silva, Á. R. D., Silva, L. L. D., Frazão, J. J., Salgado, F. H. M., Silva, M. C. D., \& Correche, 
V. (2012). Resistência mecânica à penetração do solo com diferentes coberturas vegetais sob sistema. Revista Científica Eletrônica de Agronomia, Garça, 22(2).

Silva, C. A., \& Machado, P. D. A. (2000).

Sequiestro e emissão de carbono em ecossistemas agrícolas: estratégias para o aumento dos estoques de matéria orgânica em solos tropicais. Embrapa Solos-Documentos (INFOTECA-E).
Viana, J. (2008). Determinação da densidade de solos e de horizontes cascalhentos. Embrapa Milho e Sorgo-Comunicado Técnico (INFOTECA-E).

World Health Organization. (2018). The state of food security and nutrition in the world 2018: building climate resilience for food security and nutrition. Food \& Agriculture Org. 